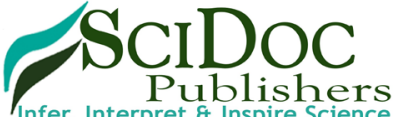

Publishers

International Journal of Clinical \& Experimental Otolaryngology (IJCEO)

ISSN 2572-732X

\title{
Ultra-High-Resolution (UHR) Optical Coherence Tomography (OCT) in the Upper Airways: Aspect of Combined High-Speed Films and UHR OCT in the Larynx
}

Research Article

Pedersen $\mathrm{M}^{*}$

ENT specialist HON. prof IBC Cambridge UK.

\section{Abstract}

\begin{abstract}
Aim: The present position paper discusses the possibilities of comparing the new ultra-high-resolution optical coherence tomography with high-speed films in order to optimize diagnosis of the phonating larynx.

Method and Results: Till now setups of OCT have had too low frequencies for evaluating the phonating larynx in the clinic. This gives artefacts on the vocal folds movements. Ultra-high-resolution optical coherence tomography is therefore discussed, possibly combined with high speed films of 2000 frames per second of the vocal folds (Richard Wolf GmbH). The UHR OCT setup is based on a supercontinuum source from NKT Photonics. The system imaging depth and maximum fields of view are $2 \mathrm{~mm}$ and $8 \mathrm{~mm}$ respectively with a inhouse constructed probe. The software Glottal analysis tools (GAT, Erlangen Germany) is used for many interesting results with highspeed films.

Discussion and Conclusion: Still OCT analyses have too low frequencies to give the real picture of the vocal fold movements to be combined with highspeed films. Till now a probe for mouth mucosa has been constructed. In the future a handhold probe for larynx combined with highspeed films has been planned.
\end{abstract}

Keywords: Ultra-High-Resolution; Optical Coherence Tomography; High-Speed Films; Larynx; Vocal Folds.

\section{Introduction}

We have earlier made a paper on OCT [1] in the clinic, because we needed some kind of evidence based objective documentation for especially pharmacological treatments. OCT might be a possibility for an office-based approach during phonation.

The main pathological changes in the larynx in the office, are in most cases of laryngeal voice disorders due to infections, allergy and reflux. The phonation is often highly compromised in these cases.Neoplasms are seldom seen in the voice clinics and they are mostly due to using the voice for a long time with the previous mentioned reasons. OCT has been used for benign and malignant neoplasms in the larynx during anesthesia for several years.

The possibility to make a exact diagnosis of functional voice disorders has been improved significantly with the high-speed setup for clinical use, that has been on the market since the beginning of this millennium (High Speed Digital Imaging equipment, 5562 ENT setup, Richard Wolf GmbH, 2006).With this equipment a slow motion video of the vocal fold movements with $2.000-4.000$ frames per second can be made, so the right and left vocal fold and the area surrounding them are seen very clearly.

A software has been developed for highspeed films (Glottal Analysis Tools (GAT), Vocal fold recording analysis software, version.8.1, University Hospital Erlangen, Medical School, 2014). With this software up to 183 calculations and measurements of video and audio signals, glottal area waveform and trajectories can be made. It is possible to reproduce the glottal area waveform and the phonovibrogram of single glottal area waves. We have not been able to statistically verify the treatment for subjective complaint of hoarseness with these measurements, not even with online videos compared with segmentation and kymography.

Therefore, we have focused on Optical Coherence Tomography (OCT), where Brian Wong and his team have made progress in

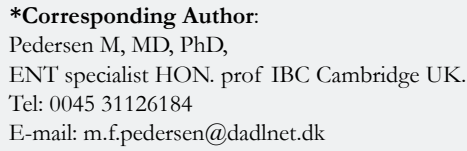

Citation: Pedersen M. Ultra-High-Resolution (UHR) Optical Coherence Tomography (OCT) in the Upper Airways: Aspect of Combined High-Speed Films and UHR OCT in the Larynx. Int J Clin Exp Otolaryngol. 2019;5(1):101-105. doi: http://dx.doi.org/10.19070/2572-732X-1900018

Copyright: Pedersen $\mathbf{M}^{\circ}$ 2019. This is an open-access article distributed under the terms of the Creative Commons Attribution License, which permits unrestricted use, distribution and reproduction in any medium, provided the original author and source are credited. 
showing the single movements of the vocal folds online in a clinical setup [2]. On the OCT it is possible to see the cellular layers of the vocal folds during phonation and also the edge. This means that it will be possible to see changes in pathology, both of the tissue layers and the margins of the vocal folds [3]. Still the speed of the film was too low, and the danger is therefore artefacts.

Another interesting aspect is a combination including hormonal aspects of OCT with other parameters of development of voice [4], OCT was made during anesthesia comparing a 2.5 year old with a 16 year old boy [5].

In the clinic based on high-speed films, we often see laryngopharyngeal reflux with more or less severe edema of the arytenoid regions and the vocal folds. It can be difficult to give a prognosis especially to people who are dependent of their voices even with highspeed films. That is why we initiated a collaboration with the Danish Technical University (Lyngby) in order to develop a ultra-high-resolution optical coherence tomography. At this university, they are experienced with OCT in other fields [6]. The technique that they use has been described recently [7].

Many authors underline the necessity of a refined OCT method to document office-based treatment effect in the larynx. Until now our UHR OCT setup can be used to detect tissue abnormalities of the oral mucosa online, that functions as well as biopsies. With this equipment it is possible to see the cellular layers in the oral mucosa in the same way as described in pathology by others [8]. In the future we hope to use UHR OCT to evaluate the larynx during phonation combined with highspeed films without artefacts.

\section{Material and Method}

A setup with UHR OCT that is capable of being combined with high-speed films with 2.000 frames per second has been constructed. The point is that the high resolution gives information of cellular layers in the larynx during phonation and can be used with functional and pathological mucosal aspects.

The OCT system applied for the study is an ultrahigh resolutions system constructed in-house. The setup is based on a supercontinuum source from NKT Photonics providing a large near-infrared band with centered at approximately 1.3 micrometers. The light is forwarded to a beam splitter after which a sample and a reference mirror are exposed. The returning combined light signal is relayed to a spectrometer (Wasatch Photonics) detecting interferometric signals in a wavelength range of 1.074-1478 micrometer. The spectra detected are resampled, dispersion compensated, and attenuation corrected. The system demonstrates an axial (depth) resolution below 3 micrometers, a lateral resolution of 6 micrometer, a sensitivity of $90 \mathrm{~dB}$ and a line rate of $76 \mathrm{kHz}$. The imaging depth and maximum field of view are $2 \mathrm{~mm}$ and $8 \mathrm{~mm}$, respectively.

For interfacing the oral mucosa, a handheld probe was constructed till now enabling imaging of the lining oral mucosa $[6,7]$. The pathology described is of great interest [8].

Results
Figure 1. High speed film scores with 4000 pictures/sec of the larynx including the arytenoid regions. Score 1 indicates a normal arytenoid region and normal vocal folds. Score 3 indicates a moderate oedema of the arytenoid region and normal vocal folds. Score 5 indicates almost total closure of the larynx due to arytenoid oedema.
(A)

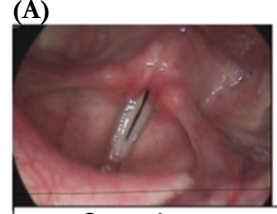

Score 1
(B)

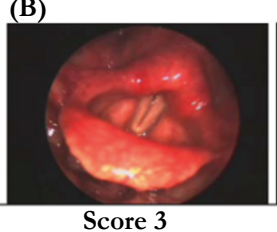

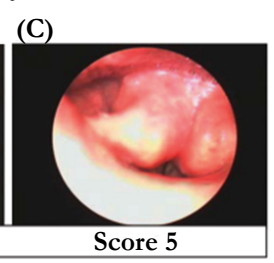

Figure 1 from ref. [1]

Figure 1 introduces the diagnostic problem of the larynx with edema of the arytenoid regions. It is impossible to evaluate with high-speed film alone, how serious the pathology of the mucosa is.

Figure 2a. A normal larynx with marking of the middle of the vocal ridge (GAT).

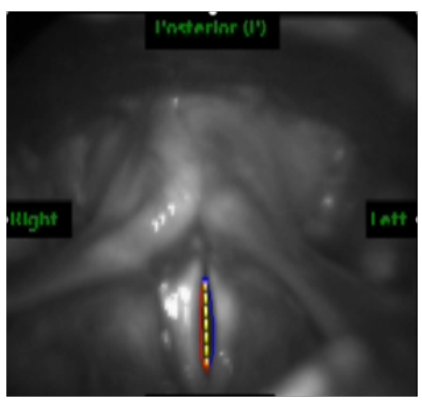

Figure $2 b$. The areas between the vocal fold, single movements during phonation.

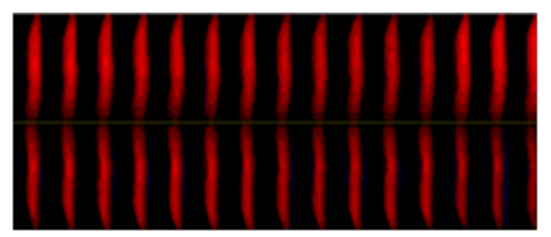

Figure 2c and Figure 2d: The software reproduction (GAT) of the vocal ridge- $d$ : Cross section of the vocal folds in time.

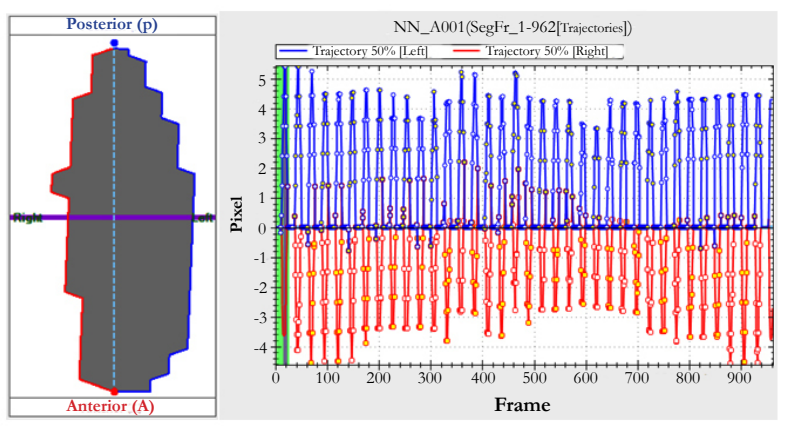

Figure 2a shows what the software Glottal Analyses Tool (GAT, University clinic Erlangen, ENT department) can. It is an overview of a normal larynx shown with high-speed film and a 
marking of the vocal ridge.

Figure $2 \mathrm{~b}$ shows the phonovibrogram with each vocal fold on top of each other, the red color is open phase between the vocal folds shown in time - each area of the duty cycles is presented.

In Figure 2c a computerized reproduction of the area between the vocal folds is seen - this is also possible to be demonstrated with a film - that could be combined with UHR OCT. Figure $2 \mathrm{~d}$ shows the basis for the computerized reproduction of the vocal movement, made with a cross section in the middle in the vocal folds single movements in time.

Figure 3a. Detailed movements with 4.000 frames per second.

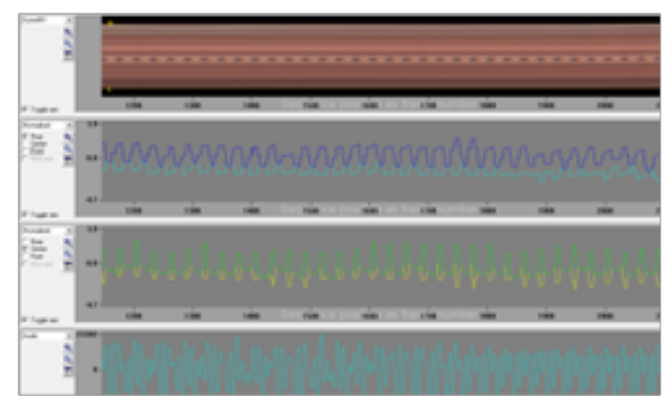

Figure $3 \mathrm{a}$ is a presentation of the segmentation made with the high-speed setup from Richard Wolf GmbH. The upper curve is a kymogram in the middle of the vocal ridge, underneath is the closure of the single vocal folds in the rear third. Then the closure of the vocal folds in the middle is presented, and in the bottom the corresponding acoustical waveform.

Figure 3b. Some of the measurements made with GAT.

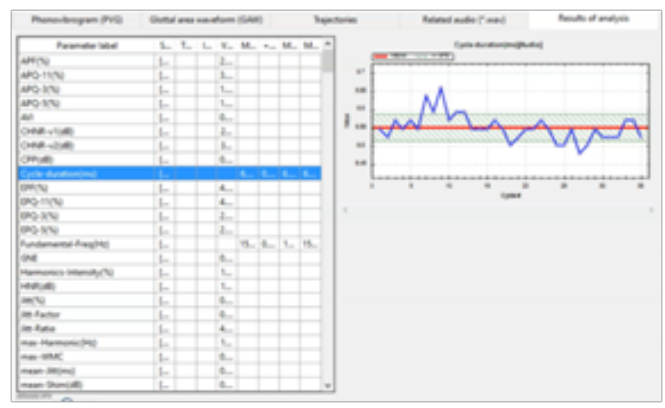

Figure $3 \mathrm{~b}$ this presentation is part of the 183 parameters that can be calculated in the glottal analysis tools, for example the variation of the fundamental frequency during measurement.

Figure 4a. Detailed measurement marking the free edges of the vocal folds with high-speed film.

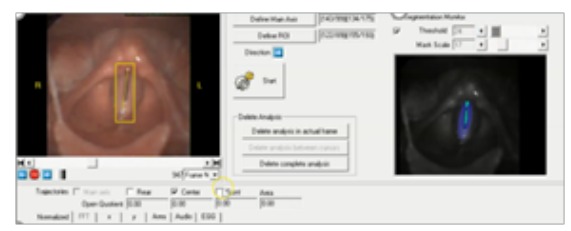

Figure 4a shows an overview of the larynx and the marking of the borders of the vocal folds during phonation using the software from Richard Wolf GmbH, the picture is from a video.

Figure $4 b$. The setup for OCT on a stiff scope from Beckman Institute California [3].

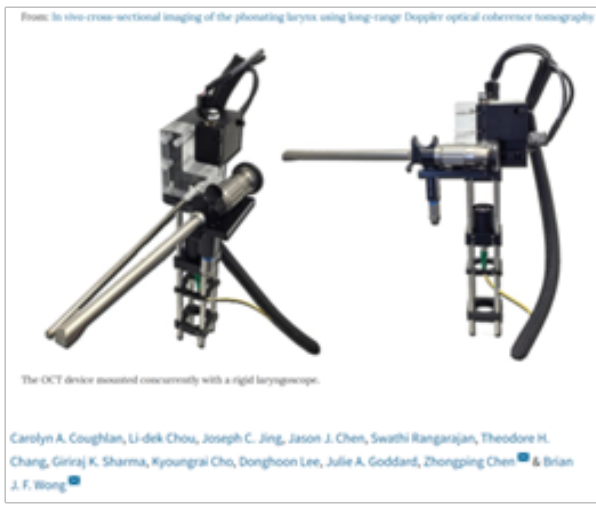

Figure 4c. One picture of a film with 200 frames per second OCT [4].

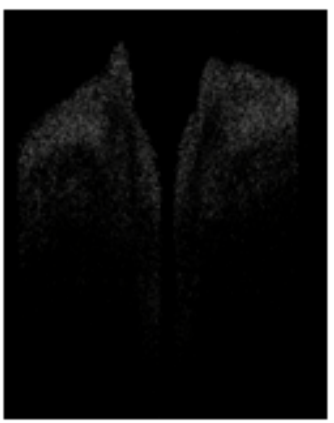

Figure $4 \mathrm{~b}$ presents the setup with a stiff light source and OCT probe with low speed, resulting in a film from which a picture seen in figure $4 \mathrm{c}$, the borderlines of the vocal ridge are presented and also the cellular layers. This is the ambition, to coordinate with high-speed films and ultrahigh resolution OCT with 2.000 frames per second.

Figure 5 a,b. We present our book on voice development combined with hormonal and pediatric development $b$ : Shows phonetograms of a pubertal boy, in the 8th grade, from the book.
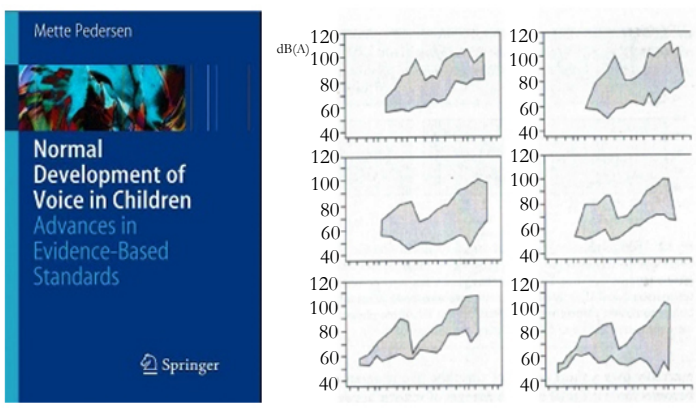

There has been many attempts to solve the problem of the diagnosis of the vocal fold layers especially during puberty. Figure $5 \mathrm{a}$ is a picture of our book on the normal development of the voice, with many acoustical measures combined with pediatric and hormonal development [4]. In Figure 5b for example the phonetograms of an 8th grade school boy is shown from the 
book.

Figure 5c. OCT during anesthesia of a 2.5 and 16 year old boy.

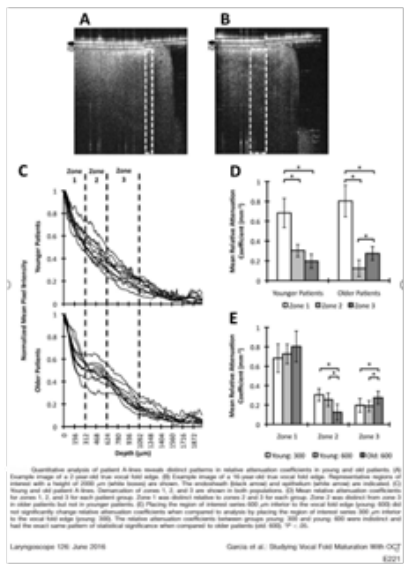

Figure $5 c$ is from Garcia et al. including the description with a algorithm of attenuation coefficient [5]. The 2.5 year old child has two layers of the vocal folds, the 16 year old has three layers. The eventual combination of office OCT with the hormonal aspects of the voice development is of great interest.

Figure 6. Acid and mucus from the stomach in the arytenoid region.

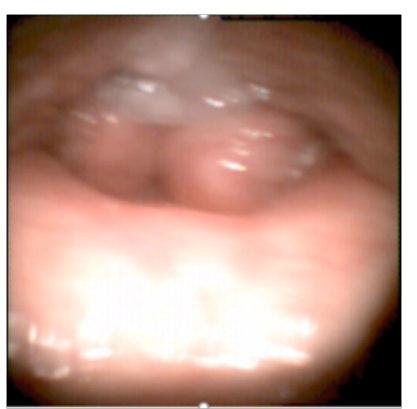

Figure 6 is part of a film of laryngopharyngeal reflux that shows how the mucus and acid comes up from the stomach and irritates the larynx in less 0.2 second in our high-speed setup with 4.000 pictures per second.

The previous figures were mostly of our high-speed film results. Until now there is no UHR OCT in function with high-speed films.

Figure 7a. The UHR OCT of the mucosal tissue in the mouth.

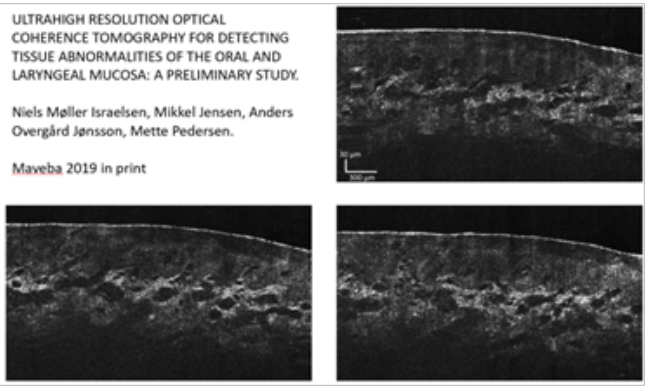

Lily Chen and Brian Wong at the Beckman Institute in California made anoffice-space setup in vivo but it is limited by sampling speed of 200 pictures per second, being under Nyquist sampling frequency resulting in motion artifacts.

Lily Chen underlines the necessity of a refined method to document treatment effect of voice disorders during phonation. This is why Niels Møller Israelsen and his team made preliminary study of UHR OCT for detecting tissue abnormalities of the oral mucosa online. 3 pictures of the mucosa of the 2 layers in the mouth shown in Figure 7a. The pathology of the oral mucosa has been nicely described [8].

Figure7b. A real biopsy from the oral mucosa (Google reprint)

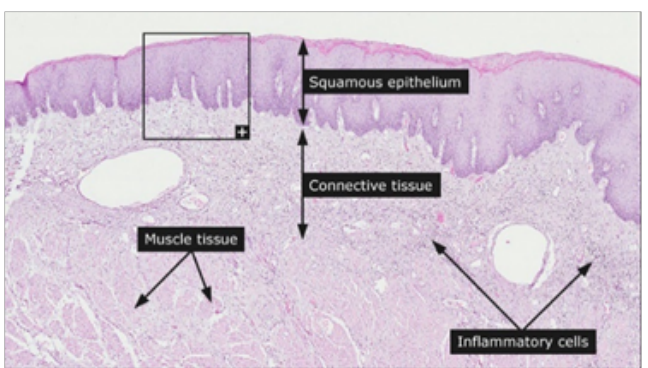

Figure $7 \mathrm{~b}$ presents a biopsy of the mucosal tissue of the mouth. Inflammatory cells are presented - thisis among the ambitions to define with our larynx setup when it is ready with UHR OCT and high-speed films.

Figure 8a. UHR OCT 6x6 mm.

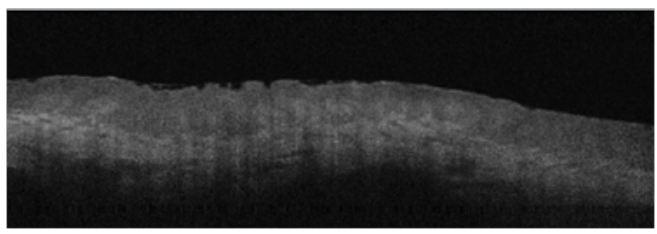

Figure 8b. 6x6 mm UHR OCT combined with OCTA overlay, to present the vascularization.

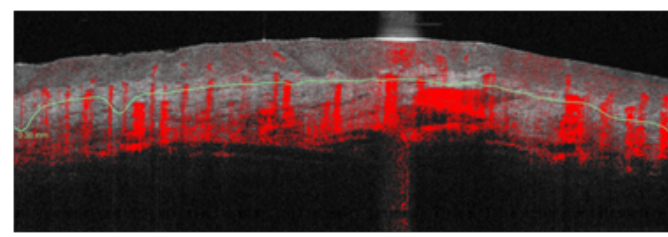

In Figure 8a 6x6 mm oral UHR OCT slice and in Figure 8b OCTA overlay in a way that the vascular picture can be seen.

Figure 8c. 3x3 UHR OCT epithelium line to the left.

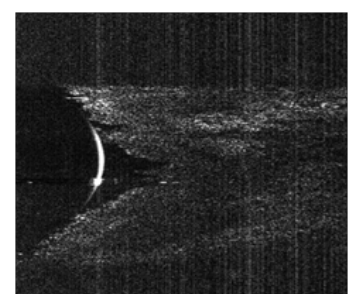


What is interesting in Figure 8c online mucosa pictures of the $3 \times 3 \mathrm{~mm}$ pictures is that the cellular layers resemble the ones in Figure 4c. That is the option to combine with the high-speed films, online during phonation with 2000 pictures per second of the vocal folds.

\section{Discussion}

The purpose of this paper was to give an overview for combining high-speed films with UHR OCT during phonation. For some time OCT has been used also in larynx disorders. OCT plays a role during anesthesia among others to get markings of the borders of benign as well as malignant neoplasms.

The problem is that the basis function, phonation, of the larynx cannot be assessed. Many sophisticated methods as presented in GAT. Airflow methods has been developed for a long time [9], this is also the case for electroglottography [10]. But they do not show difference hoarse and "non-hoarse" patients sufficiently [11].

The setup presented $[2,3]$ is of great interest. Still the problem of only 200 pictures per second is not enough to secure stability and there will be artifacts as also seen in stroboscopy. Maguluri GN et al., [12] have succeeded with online OCT on a calf ex-vivo with $50 \mathrm{hz}$.

There are many questions to be asked and many treatment aspects to be documented as referred to by others. Many technical problems have been solved, still the low speed of the OCT methods give the risk of artefacts [13]. Therefore, this research of UHR OCT combined with high-speed films is necessary also for the singing voice.

UHR OCT is an emerging technology for minimal invasive visualization of assessment of human mucosa in the upper airways, Three-dimensional imaging of the vocalizing larynx by UHR OCT is attempting to reconstruct the human larynx for visualization and kinetics [14], still the difficulty is the probe to coordinate UHR OCT with high-speed films of the moving larynx in the clinic.

\section{Conclusion}

In this presentation we described the problems for UHR OCT in the clinic with movements of the vocal folds of the larynx combined with high-speed films.

To use stroboscopy [12] gives artifacts because of lack of movement adaption, mostly only every 4 th movement is displayed. It is a step forward, the next step to understand laryngeal function during phonation also in pathology is using UHR OCT combined with high-speed films.

\section{Acknowledgement}

I am very grateful to:

Niels Israelsen from the Danish Technical University, lyngby for the UHR OCT pictures, The students Christian Larsen and Anders Overgaard Jønsson for their great help in writing the manuscript.

\section{References}

[1]. Pedersen M, Agersted A, Akram B, Mahmood S, Jønsson A, Mahmood S. Optical coherence tomography in the laryngeal arytenoid mucosa for documentation of pharmacological treatments and genetic aspects: a protocol. Advances in Cellular and Molecular Otolaryngology. 2016 Jan 1; 4(1):32246. https://doi.org/10.3402/acmo.v4.32246

[2]. Coughlan CA, Chou L, Jing JC, Chen JJ, Rangarajan S, Chang TH, et al.In vivo cross-sectional imaging of the phonating larynx using long-range Doppler optical coherence tomography. Nature Science Reports. 2016; 6: 22792. PMID: 4785353. https://doi.org/10.1038/srep22792

[3]. Chen 1, Sharma GK, Badger C, Hong E, Chou Ld, Rangarajan S, et al. Quantitative motion analysis of optical coherence tomography images in vivo human folds, World Voice Consortium Conference Copenhagen 2017.

[4]. Pedersen M. Normal development of voice in children: Advances in evidence-based standards. Springer Science \& Business Media; 2008 Sep 20. https://doi.org/10.1007/978-3-540-69359-8

[5]. Garcia JA, Benboujja F, Beaudette K, Guo R, Boudoux C, Hartnick CJ. Using attenuation coefficients from optical coherence tomography as markers of vocal fold maturation. Laryngoscope. 2016; 126(6): E218-23. PMID: 26525926

[6]. Israelsen NM, Maria M, Mogensen M, Bojesen S, Jensen M, Haedersdal M, et al. The value of ultrahigh resolution OCT in dermatology - delineating the dermo-epidermal junction, capillaries in the dermal papillae and vellus hairs. Biomed Opt Express. 2018 Apr 19; 9(5):2240-65. PMID: 29760984. https://doi.org/10.1364/BOE.9.002240

[7]. Israelsen NM, Jensen M, Jønsson AO, Pedersen M. Ultrahigh resolution optical coherence tomography for detecting tissue abnormalities of the oral and laryngeal mucosa: a preliminary study. MAVEBA Proceedings 2019. http:// maveba.dinfo.unifi.it/abstract-book/.

[8]. Wei W, Choi WJ, Men S, Song S, Wang RK. wide-field and long-rangingdepth optical coherence tomography microangiography of human oral mucosa (Conference Presentation). In Lasers in Dentistry XXIV. International Society for Optics and Photonics. 2018 Mar 14; 10473. https://doi. org/10.1117/12.2290685

[9]. Rothenberg M. Measurement of airflow in speech. J Speech Hear Res. 1977; 20(1): 155-76. PMID: 139503. https://doi.org/10.1044/jshr.2001.155

[10]. Pedersen MF. Electroglottography compared with synchronized stroboscopy in normal persons.. Folia Phoniatr (Basel). 1977; 29(3):191-9. PMID: 924308. https://doi.org/10.1159/000264088

[11]. Pedersen M, McGlashan J. Surgical versus non-surgical interventions for vocal cord nodules. Cochrane Database Syst Rev. 2012 Jun 13;(6):CD001934. PMID: 22696326. https://doi.org/10.1002/14651858.CD001934.pub2

[12]. Maguluri GN, Mehta DD, Kobler JB, Park J, Iftimia N V. Optical biopsy of vocal folds during phonation using parallel OCT (Conference Presentation). In: Alfano RR, Demos SG, Seddon AB, editors. Optical Biopsy XVII: Toward Real-Time Spectroscopic Imaging and Diagnosis. SPIE; 2019. p. 13. https://doi.org/10.1117/12.2510479

[13]. Donner S,Bleeker S, Ripken T, Ptok M, JungheimM,Krueger A. Automated working distance adjustment enables optical coherence tomography of the human larynx in awake patients. J Medical Imaging. 2015 Apr;2(2): 026003. PMID: 26158116 https://doi.org/10.1117/1.JMI.2.2.026003

[14]. Miyamoto M, Ohara A, Arai T, Koyanagi M, Watanabe I, Nakagawa H, et al. Three-dimensional imaging of vocalizing larynx by ultra-high-resolution computed tomography. Eur Arch Oto-Rhino-Laryngology. 2019; 276(11):3159-64. PMID: 31485732. https://doi.org/10.1007/s00405$019-05620-4$ 